\title{
Leveraging Compatibility and Diversity in Computational Music Mashup Creation
}

\author{
Gonçalo Bernardo \\ University of Porto, Faculty of Engineering \\ Porto, Portugal \\ up201606058@up.pt
}

\begin{abstract}
In this paper, we advance a multimodal optimization music mashup creation model for loop recombination at scale. The motivation to pursue such a model is to 1) tackle current scalability limitations in state-of-the-art (brute force) models while enforcing the 2) compatibility, i.e., recombination quality, of audio loops, and 3) a pool of diverse solutions that can accommodate personal user preferences or promote different musical styles. To this end, we adopt the Artificial Immune System (AIS) opt-aiNet algorithm to efficiently compute a population of compatible and diverse mashups from loop recombinations. Optimal mashups result from local minima in a feature space that objectively represents harmonic and rhythmic compatibility. We implemented our model as a prototype application named Mixmash-AIS, and conducted an objective evaluation that tackles three dimensions: loop recombination compatibility, mashups diversity, and computational model efficiency. The conducted evaluation compares the proposed system to a standard genetic algorithm (GA) and a brute force (BF) approach. While the GA stands as the most efficient algorithm, its poor results in terms of compatibility reinforce the primacy of the AIS opt-aiNet in efficiently finding optimal compatible loop mashups. Furthermore, the AIS opt-aiNet showed to promote a diverse mashup population, outperforming both GA or BF approaches.
\end{abstract}

\section{CCS CONCEPTS}

- Information systems $\rightarrow$ Music retrieval; • Applied computing $\rightarrow$ Sound and music computing; $\bullet$ Software and its engineering $\rightarrow$ Functional languages.

\section{KEYWORDS}

Multimodal optimization, Musical mashup, Audio processing, Artificial immune system, Music information retrieval

\section{ACM Reference Format:}

Gonçalo Bernardo and Gilberto Bernardes. 2021. Leveraging Compatibility and Diversity in Computational Music Mashup Creation. In Audio Mostly 2021 (AM '21), September 1-3, 2021, virtual/Trento, Italy. ACM, New York, NY, USA, 8 pages. https://doi.org/10.1145/3478384.3478424

Permission to make digital or hard copies of part or all of this work for personal or classroom use is granted without fee provided that copies are not made or distributed for profit or commercial advantage and that copies bear this notice and the full citation on the first page. Copyrights for third-party components of this work must be honored

For all other uses, contact the owner/author(s).

AM '21, September 1-3, 2021, virtual/Trento, Italy

(C) 2021 Copyright held by the owner/author(s)

ACM ISBN 978-1-4503-8569-5/21/09.

https://doi.org/10.1145/3478384.3478424

\author{
Gilberto Bernardes \\ Faculty of Engineering - University of Porto \& INESC TEC \\ Porto, Portugal \\ gba@fe.up.pt
}

\section{INTRODUCTION}

Music mashup creation is a composition process greatly associated with the multiple genres of electronic dance music, involving the recombination of pre-recorded musical audio [24]. The computational modeling of music mashups has been pursued in academic and industry environments in light of digital music's significant growth and the increased interest of lay-users in the mashup creation practice. In the latter scenario, computational mashup creation overcomes the need for advanced knowledge on music theory, practice, and digital signal processing.

The computational modeling of music mashup features two foremost challenges: 1) the retrieval of compatible audio from a dataset - either the search for several optimal matches or the search of musical audio matches to a target query -, and 2) audio transformations that 'force' the audio to synchronize at some attribute level, like beat alignment or pitch shifting. Our paper focuses primarily on the underlying methods of the former processing approach, which is commonly referred to as content-based retrieval within Music Information Retrieval (MIR). Its application to music mashup creation has been identified as one of the grand challenges of the community [11].

Representative state-of-the-art applications for music mashup creation can be grouped into two main categories: 1) rule-based [6, $10,14,16,26]$ and machine learning models [5]. Two prominent limitations of existing systems are their computational efficiency and adaptability. Rule-based systems adopt expensive brute-force dataset search to retrieve mashup candidates, thus imposing limitations at scale. The machine learning system requires large (and barely existing) multi-track mashup datasets to train the network. Moreover, once the machine learning network is trained, the model is somehow limited to the style of the training dataset, and little adaptation to user preferences can be accommodated.

This paper addresses the above limitations by proposing a system for assisting music mashup creation at scale from large repositories of loops. In other words, our task can be defined as a search problem that must account for 1) scalability, 2) compatibility, and 3) diversity. It ought to guarantee the adoption of large (or variable-sized loop datasets), and the search results should account for optimal recombination - according to music theory principles (e.g., low degrees of dissonance, as well as small harmonic and rhythmic distances) -, while equally enabling diverse results which accommodate personal preferences or multiple stylistic traits.

Evolutionary multimodal optimization is a class of algorithms that tackle the three above requirements. It typically embeds parallel and efficient search capabilities that optimize a given function to locate multiple diverse solutions within a search space [27]. A prominent algorithm for multimodal optimization is the Artificial 
Immune System (AIS) opt-aiNet [7]. A prominent applications of opt-aiNet to music, namely audio-content processing is computeraided musical orchestration $[1,4]$. In the latter works, an efficient optimization search is performed across a large musical instrumental note corpus to find (timbral) matching and diverse orchestrations given a reference sound.

Our work adopts opt-aiNet for searching compatible and diverse loop mashups from large loop datasets. Compatibility is driven by two main objective criteria, harmonic compatibility [21], and rhythmic compatibility [15], broadly following the metrics proposed in Mixmash [16]. Diversity accounts for the thorough and concurrent exploration for different optimal matches across the entire search space - or rather, understanding the distances between the solutions and their perceptual output. For example, resulting optimal loop recombinations can feature different key, tempo, timbre (e.g., driven from different instrumentation), and microtiming deviations (e.g., swing feeling). Conversely to existing systems driven by computationally expensive brute-force (BF) search methods (e.g., [6] and [16]), we aim to provide a computer-aided tool that enables a fluid (efficient) workflow on a large user-defined loop dataset while promoting a diverse set of optimal mashups. The model was implemented in Pure Data [19] as a prototype application named Mixmash-AIS.

We evaluate our model using three objective criteria: 1) the quality of the recombination (i.e., compatibility), the diversity of the resulting mashups, and the computational performance. The threefold criteria are compared against a standard (unimodal) Genetic Algorithm (GA) and brute-force (BF) approach, following the objective evaluation procedures proposed in [4].

The remainder of this paper is structured as follows. Section 2 surveys related work on computational mashup creation and evolutionary optimization system for musical audio recombination. Section 3 details the overview of the system, namely the audio features adopted, feature extraction, and the optimization search with opt-aiNet. Sections 4 and 5 outlines the evaluation procedure of the proposed model and the results, respectively. Finally, Section 6 presents conclusions and avenues for future work.

\section{RELATED WORK}

This section surveys related work to the problem of computational mashup creation at scale along two fundamental lines of research: computational mashup creation and evolutionary multimodal optimization. We start by describing representative systems for computational mashup creation that adopt rule-based and machine learning approaches. The rule-based system descriptions are intertwined with the adopted audio feature representations and metrics for rhythmic, harmonic, and timbre compatibility. Then, we address evolutionary multimodal optimization systems applied to audio recombination, mostly focusing on computer-aided orchestration using opt-aiNet.

\subsection{Computational Music Mashup Creation}

Early computational mashup creation systems focused on rhythmiconly features related to the temporal arrangement between two or more musical tracks $[2,12]$. Lee et al. [14] concentrated on rhythmic matching which serve tempo as an input parameter for the system and employ beat matching by stretching the beats through phase vocoder. Today, this strategy proliferates in commercial software such as Tracktor, ${ }^{1}$ Mashup 2, ${ }^{2}$ and Mixxx. ${ }^{3}$ Davies et al. [6] perform beat and downbeat tracking to align the tracks, based on two onset detection functions - kick drum and snare drum - while assuming a constant tempo and time signature across the entire duration of a musical track.

Advances in computational mashup creation pursued multiattribute systems, importantly accounting for harmony compatibility, commonly referred to as 'harmonic mixing' [2,16]. Multiple strategies have been adopted to measure harmonic compatibility: key affinity (or distances) in the circle of fifths ${ }^{4}$; cosine similarity between chromagram representations [6]; sensory dissonance [9]; and a combination of dissonance and perceptual relatedness indicators from Tonal Interval Vectors (TIV) distances [2, 16]. The latter metric has been shown to outperform remaining harmonic compatibility metrics by perceptually aligning with human judgments and by its computational efficacy, promoting harmonic mixing at scale [20].

Timbre has been addressed in computational mashup creation to balance the spectrum across multiple regions [6] or as a strategy to find audio content which occupies the same spectral region [16]. Davies et al. [6] proposed a spectral balance metric that privileges (overlapping) mashups resulting in flat spectral representations. Maçãs et al. [16] computes the cosine distance between mel-frequency cepstral coefficients (MFCC) to aid users in selecting musical loops with similar timbre for horizontal mashup creation, with varying degrees of timbral resemblance via a graph-based visualization of large corpora.

Conversely to the above systems, which broadly pursue mashups resulting from the vertical recombination of musical tracks, Harrison and Pearce [13], Lee et al. [14] extend the problem to the use of short fragments of musical audio in both vertical and horizontal dimensions. In other words, their model considers both overlapping musical audio recombination and their continuation in time.

\subsection{Evolutionary Optimisation for Musical Audio Recombination}

Evolutionary algorithms are a class of artificial intelligence methods greatly motivated by optimization processes inspired by natural phenomena, such as natural selection, species migration, bird swarms, human culture, and ant colonies [25]. Evolutionary optimization algorithms can be defined by two main criteria: modality (unimodality and multimodality) and the number of objective criteria to optimize (single- and multi-objective). The modality denotes optimization strategies that seek solutions for one local optimum (unimodal) or multiple local optima (multimodal) in a single run of the algorithm across multiple iterations. Multimodal evolutionary algorithms usually account for the population diversity, resulting from a comprehensive exploration of the search space [28].

Single and multiobjective optimization differ in terms of the objective search strategy applied. Single objective finds optimal

\footnotetext{
${ }^{1} \mathrm{https} / / /$ www.native-instruments.com/en/catalog/traktor/, last access on 20 April 2021.

${ }^{2}$ https://mashup.mixedinkey.com/, last access on 20 April, 2021.

${ }^{3}$ https://mixxx.org/, last access on 20 April, 2021.

${ }^{4}$ https://mashup.mixedinkey.com/, last access on 20 April
} 
solutions to a single objective function, whereas multiobjective accounts for problems with conflicting objectives with no single optimal solution [23]. In our work, we concentrate on multimodal and single-objective search optimization, particularly the AIS optaiNet algorithm.

De Castro and Timmis [7] presented the AIS opt-aiNet algorithm to solve multimodal function optimization problems. The algorithm can evolve a population of cells towards a set of optimal and diverse solutions to a problem. It employs immunological concepts of clonal proliferation, mutation, and repression to establish a network of inhibitors in the immune system network. In other words, opt-aiNet integrates local and global search to find optimal solutions while maintaining their diversity. Furthermore, the algorithm presents two additional important features: the automatic determination of the population size and a defined convergence criterion. Caetano et al. [4] adopted opt-aiNet to the problem of computer-aided orchestration, i.e., the search for instrumental combinations that match a reference sound by combining instrumental note samples. They showed the primacy of the method in promoting diverse solutions with optimal quality.

\section{MIXMASH-AIS}

In Mixmash-AIS, we adopt opt-aiNet to promote the computational efficient search for musical mashups resulting from the recombination of musical audio loops, ${ }^{5}$ understood in opt-aiNet's algorithmic composition as network cells. Mashups result from the combination of loops $l_{i} \in S$, where $i=\{1, \ldots, L\}$ is the index of the loop in the dataset $S$, with a total number of $L$ musical loops. A mashup $p$ is, therefore, a combination of musical loops.

Each loop $l_{i}$ is represented in a feature space where distances equate to their compatibility. The smaller the distance, the greater their compatibility. Optimal compatible mashups result from minimizing an evaluation function $E_{p}$ in the feature space. Diversity is guaranteed by pursuing a combination of local and global search in exploiting the feature space, resulting from iterative clonal mutation and selection of mashup candidates in the search space (see Fig. 1).

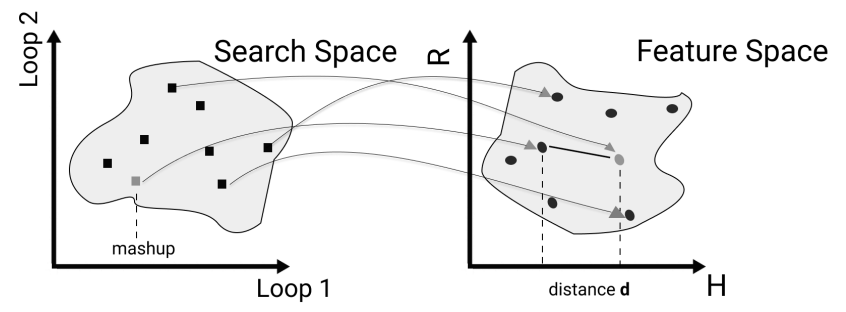

Figure 1: Representation of both search space (left) and correlated feature space (right) taking harmonic and rhythmic measurements within the loop dataset.

Fig 2 shows the architecture of Mixmash-AIS. A user-defined dataset $S$ with musical audio loops $l_{i}$ is the collection of musical audios adopted in the mashups. The feature extraction algorithm

\footnotetext{
${ }^{5} \mathrm{An}$ audio loop is a short segment of audio, e.g., a measure of a drum beat, which is created to be repeated over time [8].
}

defines harmonic $T(k)$ and rhythmic $r(b)$ representations for each dataset loop $l_{i}$, which are stored into a feature dataset. The AIS optaiNet is then adopted to search for multiple compatible and diverse mashups by evolving a random initial population. Finally, a set of optimal mashups result from overlaying the mashup component loops.

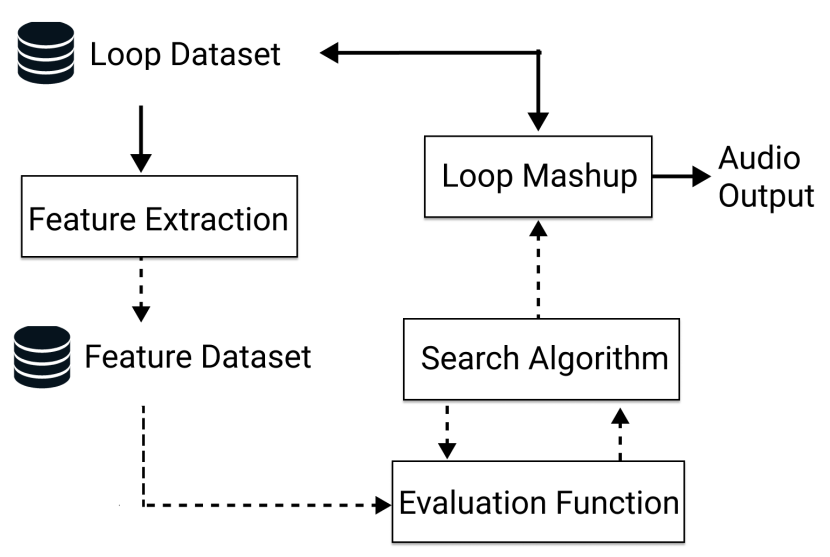

Figure 2: Multiple modules considered in Mixmash-AIS underlying our AIS computational composition process. Rectangular blocks are processing functions. Solid and dashed arrows denote audio or control flow of information between processing modules, respectively.

\subsection{Feature Extraction and Dataset}

The feature extraction module is responsible for creating two vector representations that capture the harmonic and rhythmic content of each musical audio loop $l_{i}$.

We adopt TIV, $T(k)$, as a representation for the harmonic content of an audio loop. $T(k)$ is a 12-dimensional vector computed as the DFT of a chroma vector $c(m)$, such that:

$$
\begin{aligned}
& T(k)=w_{a}(k) \sum_{m=0}^{M-1} \bar{c}(m) e \frac{-j 2 \pi k m}{M}, \\
& k \in \mathbb{Z} \quad \text { with } \bar{c}(m)=\frac{c(m)}{\sum_{m=0}^{M-1} c(m)},
\end{aligned}
$$

where $M=12$ is the dimension of the input vector and $w_{a}(k)=$ $3,8,11.5,15,14.5,7.5$ are weights derived from empirical ratings of dyads consonance used to adjust the contribution of each dimension $k$ of the DFT space [2]. We set $k$ to $1 \leq k \leq 6$ for $T(k)$, since the remaining coefficients are symmetric. $T(k)$ uses $\bar{c}(m)$ which is $c(m)$ normalised by the DC component to allow the representation and comparison of different hierarchical levels of tonal pitch, such as chords and keys, which ultimately relate to different time scales or variable duration loops.

Following Bernardes et al. [2,3], we adopt the $T(k)$ space to compute the harmonic compatibility $H$ between two given loops $l_{1}$ and $l_{2}$ using Eq. 2, which combines the dissonance $D$ and perceptual distance $P$ metrics shown in Eq. 3 and 4, respectively. The lower the values of $H$, the higher the degree of harmonic compatibility 
between two audio loops $l_{i}$. Pérez Fernández [20] has shown that the harmonic compatibility $H$ indicator perceptually captures human judgments of pleasantness to a higher degree than remaining harmonic compatibility metrics [9].

$$
\begin{gathered}
H_{l_{1}, l_{2}}=D_{l_{1}, l_{2}} \cdot P_{l_{1}, l_{2}} \\
D_{l_{1}, l_{2}}=1-\frac{a_{1} T_{1}(k)+a_{2} T_{2}(k)}{a_{1}+a_{2} w_{a}(k)}
\end{gathered}
$$

where $a_{1}$ and $a_{2}$ are the amplitudes of $T_{1}(k)$ and $T_{2}(k)$, respectively.

$$
P_{l_{1}, l_{2}}=\sqrt{\sum_{k=1}^{6}\left|T_{1}(k)-T_{2}(k)\right|^{2}}
$$

A rhythmic histogram $r(b)$ [15], where $b=60$ bins, is adopted to represent the rhythmic content of a musical loop as amplitude modulations. The representation derives from rhythmic patterns [18], a time-invariant matrix representation of loudness fluctuations in 24 critical Bark bands of the human's listening range. Their fundamental difference is that rhythmic histogram $r(b)$ accumulates all 24 critical bands onto a single bin, resulting in a vector of 60 frequency modulation bins in the $[0,600] \mathrm{BPM}$ range [15]. The motivation to adopt rhythmic histograms $r(b)$ in our work, instead of the more common rhythmic pattern representation, is to minimize pitch or spectral differences in the similarity computation, namely in light of the typical single-instrument nature of musical loops used in production settings [18, 22].

To compute a rhythmic histogram $r(b)$, we adopt a two-stage extraction process. First, we group the frequency bands by loudness sensation, using a short-time Fourier transform. The resulting spectral representation is then converted into a time-invariant 24 critical Bark bands modulation frequency spectrum by applying a second Fourier transform. High amplitudes values in the rhythmic histogram $r(b)$ denote a recurrent period in the musical audio. For example, Figure 3 shows the rhythmic histogram with four predominant peaks at multiples of the tempo.

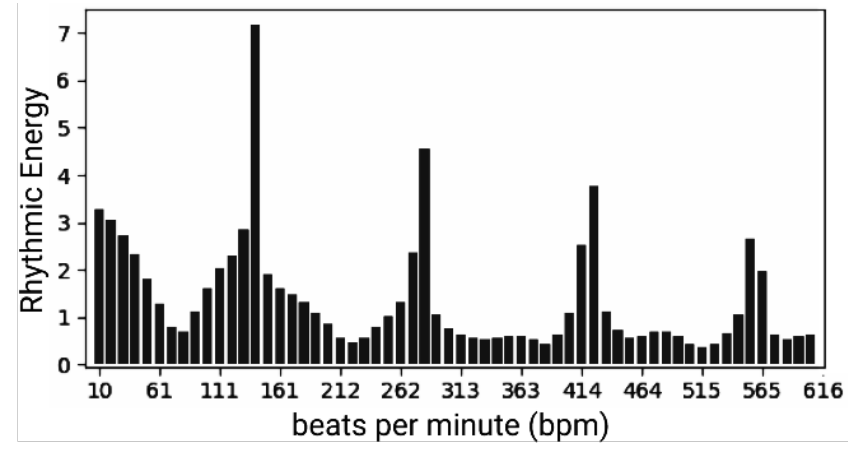

Figure 3: Rhythmic periodicity function for an audio loop including leads and brass within rhythmic pulse.

The distance between rhythmic histograms $r_{1}(b)$ and $r_{2}(b)$ from two musical loops $l_{1}$ and $l_{2}$ is computed as their angular distance, such that:

$$
R_{l_{1}, l_{2}}=\operatorname{arcos} \frac{r_{1}(b) \cdot r_{2}(b)}{\left|r_{1}(b)\right|\left|r_{2}(b)\right|} .
$$

\subsection{Evaluation Function}

To ensure high values of compatibility in a diverse space of solutions, AIS opt-aiNet assesses a population of musical loop mashups $p$ at each iteration. The population evolves across multiple iterations by minimizing an evaluation function $E_{p}$. To compute an objective evaluation value per mashup $p$, we first define $H_{p}$ and $R_{p}$ as the sum of all pairwise distances across the component loops $l_{i}$ in the mashup $p$. A total of $u(u-1) / 2$ pairwise values per harmonic and rhythmic representation are summed using Eq. 2 and 5, respectively, where $u$ is the total number of loops $l_{i}$ in a mashup $p$. Then, we apply Eq. 6 to combine the resulting harmonic $H_{p}$ and rhythmic $R_{p}$ compatibility metrics linearly. Furthermore, a high penalty is applied to mashups $p$ that include repeating loops $l_{i}$, such that:

$$
E_{p}=H_{p}+R_{p}+F_{p}
$$

where $F_{p}=0$ if no $l_{i}$ duplicate loops are found in $p$ and $F_{p}=50$, otherwise.

\subsection{Search Algorithm}

The immunological operations in AIS opt-aiNet - cloning, mutation, and affinity suppression of solution candidates - evolve an initial random population towards compatible and diverse mashups in the immune network. Maintenance of compatibility is assured by the evaluation function $E_{p}$ and leveraged by cloning and mutation operators, which optimize the population of mashup candidates across multiple regions. Valleys (or local minima) in the multimodal search space indicate optimal mashup candidates $p$. Fig 4 shows a flowchart diagram of the AIS opt-aiNet algorithm used in our work.

The AIS opt-aiNet algorithm starts by instantiating a random population of mashups $p$. The initial number of mashups in the population (i.e., population size or the number of network cells) is not relevant. The algorithm includes mechanics for the automatic adjustment of the population size via affinity suppression and population expansion. Cloning is responsible for creating a number $N_{c}$ of offspring cells per mashup in the population (or network cell) that are identical copies of their parent cell. Each clone includes the parent and its $N_{c}$ offspring. The offspring clones undergo an operation of somatic mutation to become variations of its parent. In other words, mutation asserts if a given loop $l_{i}$ in a mashup $p$ is changed. A probability of a given loop $l_{i}$ within a mashup $p$ to be mutated is inversely proportional to the mashup $p$ evaluation value. Following Caetano et al. [4], we adopt Eq. 7 to define the mutation probability of a given loop $l_{i}$ within a mashup $p$.

$$
\chi=\exp (-\gamma \hat{E}),
$$

where $\gamma=1.2$ is a constant and $\hat{E}$ is the normalized evaluation value to the $[0,1]$ range from the corresponding mashup $p$ (or to the cell undergoing mutation). For each of the loops $l_{i}$ in the mashup $p$, a random decimal value in the $[0,1]$ range will determine its mutation onto a different audio loop index $i$. If the decimal value is $\leq \chi$, another loop from the dataset $S$ is randomly fetched, or, in other words, its index $i$ is replaced by a random index number in the $[1, L]$ range.

A clonal selection performs an elitist optimization of the population to retain the best-ranked mashups per clone. To this end, all clone mashups are evaluated using Eq. 6 and the mashup with the smallest evaluation value $E_{p}$ per clone is retained in the population. 


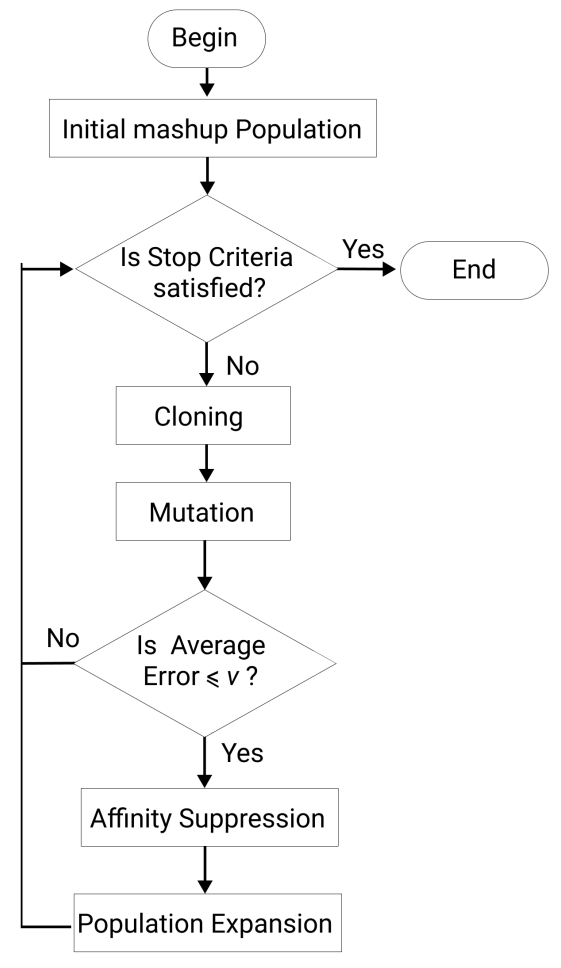

Figure 4: Opt-aiNet flowchart diagram.

Then, the population's average fitness is computed to assess if the local region optimization of the mashups has stabilized. To compute the stabilization of the population, we compute the average error of the population evaluation using Eq. 8, which computes the modulo of the ratio between the average evaluation values of the previous iteration to the current iteration subtracted from unity. If the average error is $\leq v$ where $v=0.001$, the population is said to have stabilized, and the algorithm continues to its affinity suppression of solution candidates, and population expansion. If the condition does not hold, a new iteration with a clonal selection of the population is performed.

$$
\text { Average Error }=\left|1-\frac{\text { Average of Old Evaluation }}{\text { Average of Evaluation }}\right|,
$$

To maintain diversity, AIS opt-aiNet adopts the suppression operator to excludes mashups $p$ with high affinity, or below a given distance threshold $t$ in the feature space. Pairwise mashup distances in the feature space are computed as the angular distance $A$ of the weighted combination of harmonic $T(k)$ vectors and the linear combination of rhythmic $r(b)$ vectors using Eq. 9 and 10, respectively. Prior to the angular distance computation each mashup $p$ is represented by the concatenated $\left(T_{c}(k), r_{p}(b)\right)$ vector.

$$
\begin{gathered}
T_{p}(k)=\sum_{i=1} a_{l_{i}} T_{l_{i}}(k) \\
r_{p}(b)=\sum_{i=1} r_{l_{i}}(b)
\end{gathered}
$$

Hence, suppression excludes mashups $p$ (candidate mashups) within a given radial range, retaining the local optima mashups that minimize the value of $E_{p}$ in Eq. 6 in multiple regions of the search space. By excluding similar mashups $p$ from the immune network, we ensure diversity in the population. The remaining mashups in the immune network after suppression are referred to as memory cells.

The AIS opt-aiNet includes two stopping criteria conditions. Whenever one condition is met, the iterative method is stopped, and the population is output. The system's output is the total number of mashups in the population ranked by their evaluation $E_{p}$ value in ascending order, i.e., from the best to worse ranked local optima. The stopping criteria include the user-defined maximum number of interactions $u$ or once the number of memory cells in a population has stabilized over two consecutive iterations. If the number of memory cells does not stabilize, a percentage $d=40 \%$ of random network cells is appended to the population to expand the immune network capacity to explore the space further.

Finally, once the algorithm outputs a population of local optima mashups, each mashup $p$ is synthesized by overlapping its component audio loops $l_{i}$, retrieved from the loop dataset $S$ given their index $i$.

\section{MODEL EVALUATION}

As shown in previous studies [6,9], compatibility between musical loops in a mashup is fundamental to user enjoyment. However, diversity in mashup creation is equally important in promoting multiple solutions from which users can select, taking into account their personal preferences. Therefore, an application for assisting users in mashup creation should provide multiple and perceptually different solutions.

In this context, we adopt objective measures to evaluate the 1) compatibility, 2) diversity, and 3) computational performance of the proposed model, Mixmash-AIS, compared to a standard genetic algorithm (GA), Mixmash-GA, and the original brute force (BF), Mixmash-BF applications. All systems use the same feature space, which results from the combined harmonic $H$ and rhythmic $R$ representations. More importantly, the same evaluation function in Eq. 6 to assess the compatibility of a mashup $E_{p}$.

We implemented the three prototype systems in Pure Data [19], whose code, documentation, and several mashup examples are available as supplementary material to this publication. Mixmash-GA adopts a standard GA with a uniform crossover with 0.7 probability, uniform mutation with 0.2 probability, roulette wheel selection, and elitism (top 5\% individuals). Similar to Mimxash-AIS, Mixmash-GA returns mashups ordered by fitness value.

Ideally, Mixmash-AIS ought to reach a similar level of compatibility in top-ranking mashups as the brute force Mixmash. Moreover, we expect Mixmash-AIS to outperform Mixmash-GA in terms of compatibility and diversity. Standard GAs can prematurely converge to the same local optimum, which is not guaranteed to be the global (compatible) optimum, whereas AIS opt-aiNet returns multiple (diverse) local optima.

A dataset of 551 hip-hop instrumental loops from Apple Loops which are commonly distributed with proprietary Apple Digital 
Audio Workstation software, such as Garageband and Logic ${ }^{6}$ was adopted as our dataset $S$. Included loops range between five to 24 seconds and feature diverse tempo (or bpm) and multiple instruments within a large set of spectral regions, roughly in the $[40,10000] \mathrm{Hz}$ range. We have defined the AIS opt-aiNet and GA parameters to withstand an initial population of 20 cells and a value of 200 max iterations. Specifically, for AIS opt-aiNet, the number of clone generations - for each network cell - is set to 10 , and respectively with an affinity threshold of $t=0.5$, as mentioned in Section 3.

\subsection{Evaluating Compatibility}

To objectively assess and compare the compatibility in all models under evaluation, we average the evaluation function values $E_{p}$ of the 10 best-ranked mashups, thus providing an average indicator of the model compatibility. The smaller the average compatibility value, the better it complies with the objective criteria we aim to minimize, i.e., harmonic $H$ and rhythmic $R$ compatibility, and no repeating (overlapped) loops $F$. Furthermore, in the AIS and GA models, we run the algorithms 10 times to capture the diversion in optimization convergence mechanics across multiple runs. Diversion is the optimization expected to be more clearly noticeable in the results of the GA. The AIS opt-aiNet algorithm also does not guarantee similar results with each run. However, the affinity suppression and population expansion in AIS opt-aiNet algorithm minimize this behavior.

\subsection{Evaluating Diversity}

To objectively measure diversity in the three models under evaluation, we inspect distance relations across mashups in their feature space, i.e., within the same space as the affinity is calculated. Mashup locations in the feature space reflect perceptual relations amongst the regions in which they are found. Therefore, it is possible to associate diversity in the feature space with the conception of diversity along the perceptual dimensions, correlated with adopted harmonic and rhythmic features. We propose using the average distance across all unique pairwise mashups from the 10 best-ranked evaluation set in each model. A total of 45 distance values are averaged per model.

\subsection{Evaluating Computational Performance}

The computational performance of the models is instrumental to the task of computational mashup creation at scale due to the combinatorial explosion of the loop recombination number. Depending on the size of the loop dataset $L$, defined a priori by the user by selecting the dataset, it affects the complexity of the problem. A brute-force (BF) approach in the dataset under consideration, which includes 551 loops, results in 151525 unique combinations for mashups with two overlapping loops.

The associated computational runtime cost of each iteration for the AIS and GA under evaluation can be defined as $O(L V)$, where $L$ is the current population size, and $V$ is the length of the combined rhythmic and harmonic representation vectors. The affinity suppression in AIS has an additional computational cost of $O(L 2 \mathrm{~V})$.

${ }^{6}$ https://support.apple.com/guide/logicpro/apple-loops-in-logic-pro-lgcp734a05f6/ mac, last accessed on 10 May 2021.
Table 1: AIS opt-aiNet objective evaluation.

\begin{tabular}{cccccc}
\hline $\begin{array}{c}\text { Run } \\
\text { Count } \\
(\#)\end{array}$ & $\begin{array}{c}\text { Iteration } \\
\text { Count }\end{array}$ & $\begin{array}{c}\text { CPU } \\
\text { Time } \\
(\mathrm{ms})\end{array}$ & $\begin{array}{c}\text { Cell } \\
\text { Count }\end{array}$ & $\begin{array}{c}\text { Average } \\
\text { Compatibility }\end{array}$ & $\begin{array}{c}\text { Median } \\
\text { Affinity }\end{array}$ \\
\hline 1 & 35 & 2953 & 11 & 1.368 & 1.640 \\
2 & 30 & 2593 & 12 & 1.528 & 1.639 \\
3 & 60 & 4547 & 12 & 1.353 & 1.667 \\
4 & 40 & 3250 & 13 & 1.556 & 1.655 \\
5 & 75 & 6469 & 14 & 1.228 & 1.638 \\
6 & 140 & 9749 & 14 & 1.451 & 1.613 \\
7 & 80 & 7328 & 18 & 1.323 & 1.676 \\
8 & 160 & 11984 & 12 & 1.259 & 1.602 \\
9 & 95 & 8484 & 14 & 1.370 & 1.582 \\
10 & 140 & 9625 & 12 & 1.247 & 1.529 \\
\hline Total & & & & & \\
Average & - & 6708 & - & 1.368 & 1.624 \\
\hline
\end{tabular}

The BF approach does not feature multiple iterations, and its computational cost can be defined as $O\left(L^{2} V\right)$. The population in the former AIS and GA algorithms is defined by the user. In our evaluation, we adopt a population size of 25 (previous systems [4, 17] adopt values in the $[10,30]$ range). The latter $\mathrm{BF}$ approach is equal to the total number of loops $L$ in the dataset.

These costs indicate greater computational gains when adopting the GA than the AIS, whose affinity suppression adds complexity. Both AIS and GA suggest substantial gains compared to the BF. However, the former algorithms are dependent on their ability to converge. Therefore, to assess the performance of the models in the real-case scenario of Apple Loop collection recombination, we computed the average CPU usage in milliseconds (ms) over 10 runs. Furthermore, we equally report the number of interactions and the number of population cell count at convergence, which is particularly relevant for AIS due to the dynamic behavior of its population number adaptation.

\section{RESULTS}

Tables 1, 2, and 3 present the results for the dimensions under evaluation - 1) compatibility 2) diversity 3 ) computational performance - for each AIS, GA, and BF Mixmash models. We ran the two former models 10 times to account for their variability in local optima convergence. From each run of the algorithms, results account for the 10 best-ranked mashups. Since BF performs equally at every run of the algorithm, computes all possible loop dataset combinations, we only ran the algorithm once.

By comparing the average compatibility values across the AIS (1.368) and GA (2.694) models, we can observe that the AIS outperforms the standard GA model with an expressive reduction of the compatibility value, thus being able to find local minima in the search space with smaller (and optimal) values of compatibility. These results reinforce the importance of the multimodal search in guaranteeing a comprehensive search across the search space, which typically guarantees enhanced access to global and local optima. Conversely, the population of the GA algorithm typically 
Table 2: Genetic algorithm objective evaluation.

\begin{tabular}{cccccc}
\hline $\begin{array}{c}\text { Run } \\
\text { Count } \\
(\#)\end{array}$ & $\begin{array}{c}\text { Iteration } \\
\text { Count }\end{array}$ & $\begin{array}{c}\text { CPU } \\
\text { Time } \\
(\mathrm{ms})\end{array}$ & $\begin{array}{c}\text { Cell } \\
\text { Count }\end{array}$ & $\begin{array}{c}\text { Average } \\
\text { Compatibility }\end{array}$ & $\begin{array}{c}\text { Median } \\
\text { Affinity }\end{array}$ \\
\hline 1 & 200 & 1578 & 20 & 2.921 & 0.441 \\
2 & 200 & 1422 & 20 & 2.403 & 0.356 \\
3 & 200 & 1313 & 20 & 3.072 & 0.509 \\
4 & 200 & 1422 & 20 & 2.537 & 0.226 \\
5 & 200 & 1141 & 20 & 2.644 & 0.278 \\
6 & 200 & 1000 & 20 & 2.647 & 0.103 \\
7 & 200 & 1453 & 20 & 2.610 & 0.458 \\
8 & 200 & 1891 & 20 & 2.568 & 0.398 \\
9 & 200 & 1266 & 20 & 2.780 & 0.745 \\
10 & 200 & 1797 & 20 & 2.756 & 0.865 \\
\hline Total & & & & & \\
Average & - & 1428 & - & 2.694 & 0.438 \\
\hline
\end{tabular}

Table 3: Brute force objective evaluation.

\begin{tabular}{cclccc}
\hline $\begin{array}{c}\text { Run } \\
\begin{array}{c}\text { Count } \\
(\#)\end{array}\end{array}$ & $\begin{array}{c}\text { Iteration } \\
\text { Count }\end{array}$ & $\begin{array}{l}\text { CPU } \\
\text { Time } \\
(\mathrm{ms})\end{array}$ & $\begin{array}{c}\text { Cell } \\
\text { Count }\end{array}$ & $\begin{array}{c}\text { Average } \\
\text { Compatibility }\end{array}$ & $\begin{array}{c}\text { Median } \\
\text { Affinity }\end{array}$ \\
\hline 1 & - & 151523 & - & 0.774 & 1.494 \\
\hline
\end{tabular}

converges to the same region and does not guarantee to converge to local optima. The average compatibility of the BF approach (.774) is lower than the AIS (and GA), thus presenting a set of more compatible mashups in its 10 best-ranked solutions. However, AIS is enforcing the exclusion of mashup solutions in the same region of the feature space, which denotes perceptually similar mashups. Therefore, it can be excluding compatible and perceptually similar mashups, as it only retains the optimal mashup in a surrounding affinity region. The lower median affinity value of the BA compared to the AIS reinforces this assertion, as it indicates the found 10 best-ranked mashups in the BF have smaller diversity.

The median affinity for the three AIS (1.624), GA (.4379), and BF (1.494) denote a clear advantage of the AIS in promoting diversity in the 10 best-ranked mashups as it finds mashups whose global distances in the feature space are more spread then the remaining models. The GA performs very poorly in terms of diversity as it typically converges most solutions towards a unique local optimal region in the search space. In a musical context, the smaller the affinity median value, the closer are candidate solutions throughout our musical feature space. Consequently, the mashup outputs are not as perceptually different from each other.

By comparing the computational efficiency of the three models given by the CPU time to complete a set of mashup solutions (i.e., the set of optima mashup solutions in AIS or GA and the full set of pairwise comparison in $\mathrm{BF}$ ), we clearly denote some gains in the average CPU time of GA (1428 ms) and AIS algorithm (6698 ms) compared to the CPU time of the BF (151523 ms). The GA could even be further optimized as no stopping criteria have been defined. The observation that the evaluation function $E_{p}$ needs several iterations to improve the population in the latter stages of convergence. If no diversity is required, GA clearly stands as the best optimization strategy due to its efficiency. The BF approach presents an obvious high computational cost in such a combinatorial explosion problem. We could argue that once the full set of compatible combinations is computed, we could store the results and retrieve them continuously from the set. However, this strategy would fail when adopting more than two layers or overlapping loops, as their features and distances metrics would have to be recomputed. By inspecting the diverse iteration count in the AIS opt-aiNet and the resulting average compatibility values in each run, we can denote the capacity of stopping criteria in the algorithm to assess optimal convergence conditions.

\section{CONCLUSIONS AND FUTURE WORK}

We proposed Mixmash-AIS, a multimodal music mashup optimization system for loop recombination at scale. It adopts the AIS optaiNet algorithm to leverage compatible and diverse mashups while addressing the scalability issues in existing state-of-the-art BF solutions for computational music mashup creation. In promoting a diverse set of optimal mashups, the system can account for personal preferences and different stylistic traits. An objective comparison of AIS opt-aiNet to a standard GA and BF approaches in the task under study denotes the primacy of the AIS opt-aiNet in finding local and global optimal mashups, closely matching the compatibility values of the BF approach. The AIS opt-aiNet promotes diversity to a greater degree than the GA and the BF approach. Finally, GA and AIS have significant computational performance gains compared to the BF approach.

In the future, we are planning to extend the current evaluation to perceptually assess the proposed evaluation function, $E_{p}$ in Mixmash-AIS, using a listening test where users can rate the degrees of pleasantness of resulting mashups. Furthermore, new experiments will explore the addition of timbral compatibility criteria to the evaluation function $E_{p}$ without narrowing the system's capacity to promote diverse solutions.

\section{ACKNOWLEDGMENTS}

Project "Experimentation in music in Portuguese culture: History, contexts, and practices in the $20^{\text {th }}$ and $21^{\text {st }}$ centuries" (POCI-010145-FEDER-031380) co-funded by the European Union through the Operational Program Competitiveness and Internationalization, in its ERDF component, and by national funds, through the Portuguese Foundation for Science and Technology.

\section{REFERENCES}

[1] José Abreu, Marcelo Caetano, and Rui Penha. 2016. Computer-Aided Musical Orchestration Using an Artificial Immune System. In Evolutionary and Biologically Inspired Music, Sound, Art and Design, Colin Johnson, Vic Ciesielski, João Correia, and Penousal Machado (Eds.). Springer International Publishing, 1-16.

[2] Gilberto Bernardes, Matthew EP Davies, and Carlos Guedes. 2017. A hierarchical harmonic mixing method. In International Symposium on Computer Music Multidisciplinary Research. Springer, 151-170.

[3] Gilberto Bernardes, Matthew EP Davies, and Carlos Guedes. 2017. A perceptuallymotivated harmonic compatibility method for music mixing. In Procedings of the CMMR conference. 104-115.

[4] Marcelo Caetano, Asterios Zacharakis, Isabel Barbancho, and Lorenzo J. Tardón. 2019. Leveraging diversity in computer-aided musical orchestration with an 
artificial immune system for multi-modal optimization. Swarm and Evolutionary Computation 50 (2019), 100484. https://doi.org/10.1016/j.swevo.2018.12.010

[5] Bo-Yu Chen, Jordan B. L. Smith, and Yi-Hsuan Yang. 2020. Neural Loop Combiner: Neural Network Models for Assessing the Compatibility of Loops. CoRR (2020).

[6] Matthew E. P. Davies, Philippe Hamel, Kazuyoshi Yoshii, and Masataka Goto. 2014. AutoMashUpper: automatic creation of multi-song music mashups. IEEE ACM Trans. Audio Speech Lang. Process. 22 (2014), 1726-1737.

[7] L.N. De Castro and J. Timmis. 2002. An artificial immune network for multimodal function optimization. In Proceedings of the 2002 Congress on Evolutionary Computation. CEC'02 (Cat. No.02TH8600), Vol. 1. 699-704 vol.1. https: //doi.org/10.1109/CEC.2002.1007011

[8] M. Gallagher. 2009. The Music Tech Dictionary: A Glossary of Audio-Related Terms and Technologies. Course Technology. https://books.google.pt/books?id= EfgLAAAAQBAJ

[9] Roman B. Gebhardt, Matthew E. P. Davies, and Bernhard U. Seeber. 2016. Psychoacoustic Approaches for Harmonic Music Mixing. Applied Sciences 6, 5 (2016), 136.

[10] Masataka Goto. 2002. An Audio-based Real-time Beat Tracking System for Music With or Without Drum-sounds. Fournal of New Music Research 30 (09 2002). https://doi.org/10.1076/jnmr.30.2.159.7114

[11] Masataka Goto. 2012. Grand Challenges in Music Information Research. In Dagstuhl Follow-Ups: Multimodal Music Processing, Vol. 3. Dagstuhl Publishing 217-225.

[12] Garth Griffin, YE Kim, and Douglas Turnbull. 2010. Beat-Sync-Mash-Coder: A Web Application for Real-Time Creation of Beat-Synchronous Music Mashups. In Proceedings of the International Conference on Acoustics, Speech, \& Signal Processing. 2-5.

[13] Peter M C Harrison and M. Pearce. 2020. Simultaneous Consonance in Music Perception and Composition. (2020), 216-244.

[14] Chuan-Lung Lee, Yin-Tzu Lin, Zun-Ren Yao, Feng-Yi Lee, and Ja-Ling Wu. 2015 Automatic Mashup Creation by Considering both Vertical and Horizontal Mashabilities. In Proceedings of the ISMIR. 399-405.

[15] Thomas Lidy and Andreas Rauber. 2005. Evaluation of Feature Extractors and Psycho-Acoustic Transformations for Music Genre Classification. 34-41.

[16] C. Maçãs, A. Rodrigues, G. Bernardes, and P. Machado. 2018. MixMash: A Visualisation System for Musical Mashup Creation. In 2018 22nd International Conference Information Visualisation (IV). 471-477.
[17] María Navarro-Cáceres, Marcelo Caetano, Gilberto Bernardes, and Leandro Nunes de Castro. 2019. ChordAIS: An assistive system for the generation of chord progressions with an artificial immune system. Swarm and Evolutionary Computation 50 (2019), 100543. https://doi.org/10.1016/j.swevo.2019.05.012

[18] Elias Pampalk, Andreas Rauber, and Dieter Merkl. 2002. Content-Based Organization and Visualization of Music Archives. In Proceedings of the Tenth ACM International Conference on Multimedia. Association for Computing Machinery, New York, NY, USA, 570-579.

[19] Miller Puckette. 1996. Pure Data: another integrated computer music environment. ICMC (1996), 37-41.

[20] Miguel Pérez Fernández. 2020. Harmonic compatibility for loops in electronic music. Master's thesis. Universitat Pompeu Fabra.

[21] António Ramires, G. Bernardes, M. Davies, and X. Serra. 2020. TIV.lib: an opensource library for the tonal description of musical audio. ArXiv abs/2008.11529 (2020)

[22] Andreas Rauber, Elias Pampalk, and Dieter Merkl. 2003. The SOM-enhanced JukeBox: Organization and Visualization of Music Collections Based on Perceptual Models. Journal of New Music Research 32, 2 (2003), 193-210. https: //doi.org/10.1076/jnmr.32.2.193.16745

[23] Dragan Savic. 2002. Single-objective vs. multiobjective optimisation for integrated decision support. Proceedings of the First Biennial Meeting of the International Environmental Modelling and Software Society 1 (01 2002), 7-12.

[24] John Shiga. 2007. Copy-and-Persist: The Logic of Mash-Up Culture. Critical Studies in Media Communication 24, 2 (2007), 93-114.

[25] Dan Simon. 2013. Evolutionary optimization algorithms. John Wiley \& Sons.

[26] Jordan B. L. Smith, Yuta Kawasaki, and Masataka Goto. 2019. Unmixer: An Interface for Extracting and Remixing Loops. In Proceedings of the 20th International Society for Music Information Retrieval Conference, ISMIR 2019, Arthur Flexer, Geoffroy Peeters, Julián Urbano, and Anja Volk (Eds.)

[27] Ka-Chun Wong. 2015. Evolutionary Multimodal Optimization: A Short Survey. arXiv:1508.00457 [cs.NE]

[28] Ka-Chun Wong, Chun-Ho Wu, Ricky K.P. Mok, Chengbin Peng, and Zhaolei Zhang. 2012. Evolutionary multimodal optimization using the principle of locality. Information Sciences 194 (2012), 138-170. https://doi.org/10.1016/j.ins.2011.12.016 Intelligent Knowledge-Based Models and Methodologies for Complex Information Systems. 